\title{
21. CARBON AND CARBONATE ANALYSES, LEG 27
}

\author{
Gerald W. Bode, Scripps Institution of Oceanography, La Jolla, California
}

Leg 27 sediments were analyzed for total carbon and acid-insoluble (organic) carbon using a LECO acid-base Analyzer. The 3-cc sediment samples were first dried at $105^{\circ}-110^{\circ} \mathrm{C}$ and then ground to a homogeneous powder. The ground sediment was redried and two samples, a $0.1-\mathrm{g}$ and a $0.5-\mathrm{g}$ sample, were then weighed into LECO clay crucibles. The $0.5-\mathrm{g}$ sample was acidified with diluted hydrochloric acid and washed with distilled water. The sample was then dried and analyzed for acid-insoluble carbon, listed in the table as "organic" carbon. The 0.1-g sample was analyzed for total carbon without further treatment. If the result showed less than $10 \% \mathrm{CaCO}_{3}$, an additional $0.5 \mathrm{-g}$ sample was analyzed for greater accuracy. The calcium carbon percentages were calculated as follows: (\% total C-\% organic C) $\times 8.33=\% \mathrm{CaCO}_{3}$. Although other carbonates may be present, all acid-soluble carbon was calculated as calcium carbonate. All results are given in weight percent.

Detailed descriptions of the technique and theory may be found in Bader, Gerard, et al. (1970) and Boyce and Bode (1972).

For control purposes a standard sediment as made up from Deep Sea Drilling material and analyzed for total carbon at predetermined intervals with the regular samples. Listed below is the statistical data for this standard.

\begin{tabular}{ccccc}
\hline $\begin{array}{c}\text { DSDP } \\
\text { Std. }\end{array}$ & $\begin{array}{c}\text { No. of } \\
\text { Samples }\end{array}$ & $\begin{array}{c}\text { Total Carbon } \\
\text { as } \% \mathrm{CaCO}_{3}\end{array}$ & $\begin{array}{c}\text { Standard } \\
\text { Deviation }\end{array}$ & $\begin{array}{c}\text { Maximum } \\
\text { Range }\end{array}$ \\
\hline 2 & 67 & 80.0 & $0.8 \%$ & $2.1 \%$ \\
\hline
\end{tabular}

These data indicate the precision of the mechanical aspect of the LECO analysis and do not necessarily reflect the precision of the total analytical procedure, which may be affected by factors such as sampling techniques and contamination during sampling and sample preparation.

\section{REFERENCES}

Bader, R. G., Gerard, R. D., et al., 1970. Initial Reports of the Deep Sea Drilling Project, Volume 4: Washington (U.S. Government Printing Office).

Boyce, R. E. and Bode, G. W., 1972. Carbon and carbonate analyses, Leg 9, Deep Sea Drilling Project. In Hays, J. D., et al. Initial Reports of the Deep Sea Drilling Project, Volume 9: Washington (U.S. Government Printing Office), p. 747.
TABLE 1

Carbon and Carbonate Analysis

\begin{tabular}{|c|c|c|c|c|}
\hline $\begin{array}{l}\text { Core, Section, } \\
\text { Top of Interval } \\
\text { (cm) }\end{array}$ & $\begin{array}{l}\text { Subbottom } \\
\text { Depth } \\
\text { (m) }\end{array}$ & $\begin{array}{c}\text { Total } \\
\text { Carbon } \\
(\%)\end{array}$ & $\begin{array}{l}\text { Organic } \\
\text { Carbon }\end{array}$ & $\mathrm{CaCO}_{3}$ \\
\hline \multicolumn{5}{|l|}{ Site 259} \\
\hline $1-3,24$ & 3.2 & 9.5 & 0.2 & 77 \\
\hline $1-5,24$ & 6.2 & 9.0 & 0.1 & 74 \\
\hline $3-1,24$ & 17.7 & 0.1 & 0.1 & 0 \\
\hline $4-1,24$ & 27.2 & 0.2 & 0.1 & 1 \\
\hline $4-3,24$ & 30.2 & 3.2 & 0.1 & 26 \\
\hline $4-5,24$ & 33.2 & 5.1 & 0.1 & 41 \\
\hline $5-2,24$ & 38.2 & 4.7 & 0.0 & 39 \\
\hline $5-3,24$ & 39.7 & 4.6 & 0.1 & 37 \\
\hline $5-5,24$ & 42.7 & 7.3 & 0.1 & 60 \\
\hline $6-1,24$ & 46.2 & 7.2 & 0.1 & 59 \\
\hline $6-3,24$ & 49.2 & 8.9 & 1.8 & 59 \\
\hline $6-5,24$ & 52.2 & 8.9 & 1.1 & 64 \\
\hline $7-1,24$ & 55.7 & 7.4 & 0.1 & 61 \\
\hline $7-3,24$ & 58.7 & 7.1 & 0.8 & 52 \\
\hline $7-5,24$ & 61.7 & 0.2 & 0.1 & 1 \\
\hline $8-1,24$ & 65.2 & 0.2 & 0.1 & 1 \\
\hline $8-3,24$ & 68.2 & 0.2 & 0.1 & 0 \\
\hline $8-5,24$ & 71.2 & 0.1 & 0.1 & 1 \\
\hline $9-1,24$ & 74.7 & 0.1 & 0.2 & 0 \\
\hline $9-3,24$ & 77.7 & 0.1 & 0.1 & 0 \\
\hline $9-5,24$ & 80.7 & 0.1 & 0.1 & 0 \\
\hline $10-1,74$ & 84.7 & 0.1 & 0.1 & 0 \\
\hline $10-3,24$ & 87.2 & 0.1 & 0.1 & 0 \\
\hline $10-5,24$ & 90.2 & 0.1 & 0.1 & 0 \\
\hline $11-1,104$ & 94.5 & 0.1 & 0.1 & 0 \\
\hline $11-3,24$ & 96.7 & 0.2 & 0.1 & 0 \\
\hline $11-5,24$ & 99.7 & 0.9 & 0.1 & 7 \\
\hline $12-1,24$ & 103.2 & 1.1 & 0.1 & 8 \\
\hline $12-3,24$ & 106.2 & 1.0 . & 0.1 & 7 \\
\hline $12-5,24$ & 109.2 & 0.9 & 0.1 & 7 \\
\hline $12-6,115$ & 111.7 & 1.4 & 0.1 & 11 \\
\hline $13-1,24$ & 112.7 & 2.2 & 0.1 & 18 \\
\hline $13-3,24$ & 115.7 & 2.0 & 0.1 & 16 \\
\hline $13-5,24$ & 118.7 & 2.0 & 0.1 & 16 \\
\hline $14-1,24$ & 122.2 & 2.6 & 0.1 & 21 \\
\hline $14-3,24$ & 125.2 & 2.8 & 0.1 & 22 \\
\hline $14-5,24$ & 128.2 & 2.8 & 0.1 & 22 \\
\hline $15-1,24$ & 131.7 & 3.5 & 0.2 & 27 \\
\hline $15-3,24$ & 134.7 & 3.3 & 0.2 & 26 \\
\hline $15-5,24$ & 137.7 & 3.9 & 0.1 & 31 \\
\hline $16-1,88$ & 141.9 & 3.9 & 0.1 & 32 \\
\hline $16-3,24$ & 144.2 & 4.8 & 0.1 & 39 \\
\hline $17-1,85$ & 151.4 & 3.5 & 0.1 & 28 \\
\hline $17-3,24$ & 153.7 & 4.1 & 0.1 & 33 \\
\hline $18-1,114$ & 161.1 & 0.2 & 0.1 & 0 \\
\hline $18-3,24$ & 163.2 & 0.9 & 0.8 & 1 \\
\hline $19-1,24$ & 169.7 & 0.3 & 0.3 & 0 \\
\hline $19-3,24$ & 172.7 & 0.4 & 0.3 & 0 \\
\hline $19-5,24$ & 175.7 & 0.4 & 0.4 & 0 \\
\hline $20-1,24$ & 179.2 & 0.4 & 0.4 & 0 \\
\hline $20-3,24$ & 182.2 & 0.6 & 0.5 & 1 \\
\hline $20-5,24$ & 185.2 & 0.6 & 0.6 & 0 \\
\hline $21-1, .24$ & 188.7 & 0.7 & 0.6 & 1 \\
\hline
\end{tabular}


TABLE 1 - Continued

\begin{tabular}{|c|c|c|c|c|}
\hline $\begin{array}{l}\text { Core, Section, } \\
\text { Top of Interval } \\
(\mathrm{cm})\end{array}$ & $\begin{array}{l}\text { Subbottom } \\
\text { Depth } \\
\text { (m) }\end{array}$ & $\begin{array}{c}\text { Total } \\
\text { Carbon } \\
(\%)\end{array}$ & $\begin{array}{l}\text { Organic } \\
\text { Carbon }\end{array}$ & $\mathrm{CaCO}_{3}$ \\
\hline $21-3,24$ & 191.7 & 0.5 & 0.2 & 2 \\
\hline $21-5,84$ & 195.3 & 0.5 & 0.0 & 5 \\
\hline $22-1,24$ & 198.2 & 0.5 & 0.4 & 0 \\
\hline $23-1,24$ & 207.7 & 0.4 & 0.4 & 0 \\
\hline $23-3,24$ & 210.7 & 0.7 & 0.6 & 1 \\
\hline $23-5,24$ & 213.7 & 0.5 & 0.4 & 1 \\
\hline $24-1,24$ & 217.2 & 0.4 & 0.2 & 1 \\
\hline $24-3,24$ & 220.2 & 1.3 & 0.4 & 7 \\
\hline $25-1,24$ & 226.7 & 0.3 & 0.2 & 0 \\
\hline $25-3,24$ & 229.7 & 0.5 & 0.3 & 2 \\
\hline $25-5,24$ & 232.7 & 0.6 & 0.4 & 1 \\
\hline $26-1,24$ & 236.2 & 0.5 & 0.4 & 1 \\
\hline $26-3,24$ & 239.2 & 0.4 & 0.3 & 0 \\
\hline $26-5,25$ & 242.3 & 0.3 & 0.2 & 1 \\
\hline $27-1,24$ & 245.7 & 0.4 & 0.3 & 1 \\
\hline $27-3,24$ & 248.7 & 0.3 & 0.3 & 1 \\
\hline $27-5,39$ & 251.9 & 0.3 & 0.3 & 0 \\
\hline $28-1,24$ & 255.2 & 0.4 & 0.3 & 0 \\
\hline $28-3,24$ & 258.2 & 0.4 & 0.4 & 0 \\
\hline $28-5,24$ & 261.2 & 0.4 & 0.3 & 1 \\
\hline $29-1,24$ & 264.7 & 0.6 & 0.5 & 1 \\
\hline $29-3,34$ & 267.8 & 0.5 & 0.5 & 0 \\
\hline $30-1,24$ & 274.2 & 0.4 & 0.4 & 0 \\
\hline $30-3,10$ & 277.1 & 0.3 & 0.3 & 0 \\
\hline $30-5,24$ & 280.2 & 0.4 & 0.4 & 0 \\
\hline $31-1,24$ & 283.7 & 0.3 & 0.3 & 0 \\
\hline $31-3,24$ & 286.7 & 0.7 & 0.6 & 1 \\
\hline $32-1,24$ & 293.2 & 0.5 & 0.5 & 0 \\
\hline $33-1,24$ & 302.7 & 4.3 & 0.4 & 32 \\
\hline \multicolumn{5}{|l|}{ Site 260} \\
\hline $1-1,24$ & 0.2 & 7.9 & 0.1 & 65 \\
\hline $1-3,29$ & 3.3 & 0.8 & 0.2 & 5 \\
\hline $2-3,32$ & 47.3 & 10.8 & 0.1 & 89 \\
\hline $3-1,140$ & 92.9 & 0.2 & 0.2 & 0 \\
\hline $3-3,24$ & 94.7 & 0.2 & 0.2 & 0 \\
\hline $3-5,24$ & 97.7 & 10.4 & 0.1 & 86 \\
\hline $4-1,24$ & 129.7 & 1.9 & 0.5 & 11 \\
\hline $4-5,24$ & 135.7 & 4.5 & 0.5 & 33 \\
\hline $5-1,24$ & 158.2 & 0.2 & 0.1 & 1 \\
\hline $6-1,47$ & 177.5 & 5.3 & 0.1 & 43 \\
\hline $7-1,24$ & 196.2 & 0.1 & 0.1 & 0 \\
\hline $7-3,24$ & 199.2 & 0.1 & 0.1 & 0 \\
\hline $7-5,24$ & 202.2 & 0.1 & 0.1 & 0 \\
\hline $8-5,76$ & 221.8 & 0.1 & 0.1 & 0 \\
\hline $10-1,76$ & 244.3 & 7.0 & 0.1 & 58 \\
\hline $11-1,24$ & 253.2 & 6.6 & 0.1 & 54 \\
\hline $12-1,38$ & 262.9 & 4.6 & 0.1 & 38 \\
\hline $13-1,60$ & 272.6 & 1.3 & 0.1 & 10 \\
\hline $14-1,94$ & 282.4 & 0.1 & 0.1 & 0 \\
\hline $15-3,24$ & 294.2 & 0.4 & 0.2 & 2 \\
\hline $16-1,75$ & 301.3 & 0.4 & 0.1 & 2 \\
\hline $17-1,130$ & 311.3 & 3.2 & 0.1 & 26 \\
\hline $18-1,103$ & 320.5 & 2.9 & 0.1 & 23 \\
\hline
\end{tabular}

Site 261

\begin{tabular}{lrrrr}
$3-1,102$ & 48.5 & 7.7 & 0.1 & 64 \\
$3-3,24$ & 50.7 & 6.1 & 0.3 & 48 \\
$4-1,92$ & 95.9 & 8.9 & 0.2 & 73 \\
$5-1,69$ & 162.2 & 0.1 & 0.1 & 0 \\
$6-1,24$ & 171.2 & 0.1 & 0.1 & 0 \\
$6-3,24$ & 174.2 & 0.1 & 0.1 & 0 \\
$6-5,24$ & 177.2 & 0.1 & 0.1 & 0 \\
$7-1,24$ & 180.7 & 0.1 & 0.1 & 0 \\
$7-3,24$ & 183.7 & 0.1 & 0.1 & 0 \\
$8-3,24$ & 193.2 & 0.1 & 0.0 & 0 \\
$8-5,24$ & 196.2 & 0.1 & 0.1 & 0 \\
$9-2,24$ & 201.2 & 0.1 & 0.1 & 0 \\
$9-3,24$ & 202.7 & 0.1 & 0.1 & 0 \\
\hline
\end{tabular}

TABLE 1 - Continued

\begin{tabular}{|c|c|c|c|c|}
\hline $\begin{array}{l}\text { Core, Section } \\
\text { Top of Interval } \\
\quad(\mathrm{cm})\end{array}$ & $\begin{array}{l}\text { Subbottom } \\
\text { Depth } \\
\text { (m) }\end{array}$ & $\begin{array}{c}\text { Total } \\
\text { Carbon } \\
(\%)\end{array}$ & $\begin{array}{l}\text { Organic } \\
\text { Carbon }\end{array}$ & $\mathrm{CaCO}_{3}$ \\
\hline $12-2,50$ & 230.0 & 0.5 & 0.4 & 1 \\
\hline $14-1,24$ & 247.2 & 0.1 & 0.1 & 0 \\
\hline $15-1,10$ & 256.6 & 1.3 & 1.4 & 0 \\
\hline $16-1,93$ & 266.9 & 0.4 & 0.3 & 0 \\
\hline $19-1,121$ & 305.2 & 0.2 & 0.2 & 0 \\
\hline $19-2,24$ & 305.7 & 0.3 & 0.3 & 0 \\
\hline $19-3,27$ & 307.3 & 0.2 & 0.2 & 0 \\
\hline $19-4,24$ & 308.7 & 0.2 & 0.1 & 0 \\
\hline $19-5,24$ & 310.2 & 0.2 & 0.1 & 0 \\
\hline $20-2,24$ & 315.2 & 0.2 & 0.2 & 0 \\
\hline $21-1,54$ & 333.0 & 0.1 & 0.1 & 0 \\
\hline $21-3,143$ & 336.9 & 0.2 & 0.1 & 1 \\
\hline $22-1,24$ & 342.2 & 0.1 & 0.1 & 0 \\
\hline $22-3,24$ & 345.2 & 0.2 & 0.1 & 0 \\
\hline $22-5,24$ & 348.2 & 0.2 & 0.2 & 0 \\
\hline $23-1,24$ & 361.2 & 0.2 & 0.2 & 0 \\
\hline $23-3,24$ & 364.2 & 0.3 & 0.3 & 0 \\
\hline $24-1,69$ & 380.7 & 0.3 & 0.2 & 0 \\
\hline $25-3,24$ & 402.2 & 0.2 & 0.2 & 0 \\
\hline $26-1,29$ & 418.3 & 0.3 & 0.2 & 1 \\
\hline $27-1,25$ & 437.3 & 0.1 & 0.1 & 0 \\
\hline $28-1,83$ & 447.3 & 3.5 & 0.1 & 28 \\
\hline $28-3,24$ & 449.7 & 0.1 & 0.0 & 1 \\
\hline $29-2,24$ & 467.2 & 0.1 & 0.0 & 1 \\
\hline $29-3,24$ & 468.7 & 0.4 & 0.1 & 3 \\
\hline $30-2,24$ & 486.2 & 2.7 & 0.2 & 21 \\
\hline $30-3,24$ & 487.7 & 4.1 & 0.1 & 34 \\
\hline $31-2,24$ & 505.2 & 0.4 & 0.1 & 3 \\
\hline $31-3,24$ & 506.7 & 1.6 & 0.1 & 13 \\
\hline $31-4,24$ & 508.2 & 0.3 & 0.1 & 2 \\
\hline $31-5,24$ & 509.7 & 0.1 & 0.1 & 0 \\
\hline $32-2,24$ & 524.2 & 0.7 & 0.1 & 6 \\
\hline $32-3,24$ & 525.7 & 1.3 & 0.1 & 10 \\
\hline \multicolumn{5}{|l|}{ Site 262} \\
\hline $1-3,24$ & 3.2 & 6.3 & 0.6 & 47 \\
\hline $2-3,24$ & 8.2 & 6.2 & 1.1 & 43 \\
\hline $3-3,24$ & 17.7 & 5.2 & 1.2 & 33 \\
\hline $3-5,10$ & 20.6 & 5.4 & 1.3 & 34 \\
\hline $4-3,24$ & 27.2 & 5.1 & 1.3 & 32 \\
\hline $5-1,49$ & 34.0 & 4.1 & 1.2 & 24 \\
\hline $5-3,24$ & 36.7 & 5.4 & 1.4 & 33 \\
\hline $5-5,24$ & 39.7 & 5.0 & 1.1 & 33 \\
\hline $6-1,24$ & 43.2 & 5.4 & 1.3 & 33 \\
\hline $6-3,24$ & 46.2 & 5.7 & 1.3 & 37 \\
\hline $6-5,24$ & 49.2 & 5.7 & 1.3 & 37 \\
\hline $7-3,81$ & 56.3 & 5.6 & 1.3 & 36 \\
\hline $7-5,24$ & 58.7 & 5.4 & 1.3 & 34 \\
\hline $8-1,24$ & 62.2 & 4.8 & 1.1 & 31 \\
\hline $8-3,24$ & 65.2 & 5.1 & 1.2 & 33 \\
\hline $8-5,24$ & 68.2 & 5.2 & 1.1 & 34 \\
\hline $9-1,75$ & 72.3 & 5.0 & 1.0 & 34 \\
\hline $9-3,24$ & 74.7 & 5.2 & 1.1 & 34 \\
\hline $9-5,24$ & 77.7 & 4.9 & 1.1 & 31 \\
\hline $10-1,24$ & 81.2 & 4.7 & 1.0 & 31 \\
\hline $10-3,24$ & 84.2 & 4.5 & 1.0 & 30 \\
\hline $10-5,24$ & 87.2 & 9.3 & 0.3 & 74 \\
\hline $11-1,24$ & 90.7 & 4.1 & 0.7 & 28 \\
\hline $11-3,24$ & 93.7 & 4.4 & 0.9 & 29 \\
\hline $11-5,34$ & 96.8 & 4.9 & 0.8 & 34 \\
\hline $12-1,24$ & 100.2 & 4.4 & 0.9 & 30 \\
\hline $12-3,24$ & 103.2 & 9.1 & 0.3 & 74 \\
\hline $12-5,19$ & 106.2 & 4.2 & 0.8 & 28. \\
\hline $13-1,22$ & 109.7 & 4.7 & 1.0 & 31 \\
\hline $13-3,24$ & 112.7 & 4.5 & 1.0 & 29 \\
\hline $13-5,24$ & 115.7 & 3.9 & 0.8 & 26 \\
\hline $14-1,23$ & 119.2 & 4.6 & 0.9 & 30 \\
\hline $14-3,24$ & 122.2 & 4.4 & 0.9 & 29 \\
\hline $14-5,24$ & 125.2 & 4.7 & 0.9 & 32 \\
\hline
\end{tabular}


TABLE 1 - Continued

\begin{tabular}{|c|c|c|c|c|}
\hline $\begin{array}{l}\text { Core, Section, } \\
\text { Top of Interval } \\
\quad(\mathrm{cm})\end{array}$ & $\begin{array}{l}\text { Subbottom } \\
\text { Depth } \\
\text { (m) }\end{array}$ & $\begin{array}{l}\text { Total } \\
\text { Carbon } \\
(\%)\end{array}$ & $\begin{array}{l}\text { Organic } \\
\text { Carbon }\end{array}$ & $\mathrm{CaCO}_{3}$ \\
\hline $15-1,24$ & 128.7 & 4.6 & 0.8 & 32 \\
\hline $15-3,24$ & 131.7 & 4.4 & 0.7 & 30 \\
\hline $15-5,24$ & 134.7 & 4.7 & 0.8 & 32 \\
\hline $16-1,24$ & 138.2 & 4.4 & 0.8 & 30 \\
\hline $16-3,24$ & 141.2 & 4.3 & 0.8 & 30 \\
\hline $16-5,24$ & 144.2 & 4.1 & 0.7 & 29 \\
\hline $17-1,24$ & 147.7 & 5.4 & 0.9 & 37 \\
\hline $17-3,24$ & 150.7 & 5.3 & 1.3 & 34 \\
\hline $17-5,24$ & 153.7 & 5.3 & 0.4 & 40 \\
\hline $18-1,24$ & 157.2 & 4.5 & 1.0 & 29 \\
\hline $18-3,24$ & 160.2 & 4.7 & 1.0 & 32 \\
\hline $18-5,24$ & 163.2 & 4.5 & 1.0 & 30 \\
\hline $19-1,24$ & 166.7 & 4.5 & 0.8 & 31 \\
\hline $19-3,24$ & 169.7 & 4.5 & 0.8 & 31 \\
\hline $19-5,24$ & 172.7 & 4.5 & 0.9 & 30 \\
\hline $20-1,24$ & 176.2 & 4.5 & 0.6 & 32 \\
\hline $20-3,24$ & 179.2 & 4.3 & 0.6 & 31 \\
\hline $20-5,24$ & 182.2 & 5.1 & 0.6 & 37 \\
\hline $21-1,24$ & 185.7 & 4.7 & 0.8 & 33 \\
\hline $21-3,24$ & 188.7 & 5.6 & 1.0 & 38 \\
\hline $21-5,24$ & 191.7 & 5.0 & 0.9 & 34 \\
\hline $22-3,24$ & 198.2 & 4.3 & 0.7 & 30 \\
\hline $22-5,24$ & 201.2 & 4.6 & 0.7 & 32 \\
\hline $23-3,24$ & 207.7 & 5.6 & 0.5 & 42 \\
\hline $23-5,24$ & 210.7 & 5.6 & 0.9 & 39 \\
\hline $24-3,24$ & 217.2 & 5.1 & 0.8 & 36 \\
\hline $24-5,24$ & 220.2 & 5.1 & 0.7 & 36 \\
\hline $24-1,24$ & 214.2 & 5.1 & 0.8 & 36 \\
\hline $25-3,24$ & 226.7 & 5.4 & 1.0 & 37 \\
\hline $25-5,24$ & 229.7 & 5.7 & 0.9 & 40 \\
\hline $26-1,24$ & 233.2 & 6.1 & 0.7 & 45 \\
\hline $26-3,24$ & 236.2 & 5.6 & 0.7 & 41 \\
\hline $26-5,24$ & 239.2 & 6.5 & 0.7 & 48 \\
\hline $27-1,24$ & 242.7 & 5.8 & 0.9 & 41 \\
\hline $27-3,24$ & 245.7 & 5.9 & 0.7 & 43 \\
\hline $27-5,24$ & 248.7 & 6.4 & 0.7 & 47 \\
\hline $29-1,24$ & 261.7 & 8.2 & 0.8 & 62 \\
\hline $29-3,24$ & 264.7 & 8.2 & 0.4 & 65 \\
\hline $29-5,24$ & 267.7 & 7.8 & 0.7 & 59 \\
\hline $30-1,24$ & 271.2 & 7.6 & 0.7 & 58 \\
\hline $30-3,24$ & 274.2 & 8.7 & 0.6 & 67 \\
\hline $30-5,24$ & 277.2 & 8.5 & 0.8 & 64 \\
\hline $31-1,109$ & 281.6 & 8.6 & 0.5 & 67 \\
\hline $31-3,24$ & 283.7 & 7.9 & 0.5 & 61 \\
\hline $31-5,24$ & 286.7 & 8.4 & 0.6 & 65 \\
\hline $32-1,24$ & 290.2 & 9.3 & 0.9 & 70 \\
\hline $32-3,24$ & 293.2 & 8.2 & 0.8 & 62 \\
\hline $32-5,24$ & 296.2 & 8.7 & 0.6 & 68 \\
\hline $33-1,44$ & 299.9 & 8.9 & 0.9 & 67 \\
\hline $33-3,24$ & 302.7 & 8.4 & 0.9 & 63 \\
\hline $33-5,24$ & 305.7 & 8.4 & 1.0 & 62 \\
\hline $34-1,24$ & 309.2 & 8.7 & 0.9 & 65 \\
\hline $34-3,24$ & 312.2 & 8.6 & 0.9 & 64 \\
\hline $35-1,53$ & 319.0 & 9.3 & 0.7 & 72 \\
\hline $35-3,24$ & 321.7 & 8.8 & 0.9 & 66 \\
\hline $35-5,24$ & 324.7 & 8.2 & 0.7 & 62 \\
\hline $36-1,24$ & 328.2 & 6.8 & 1.0 & 48 \\
\hline $36-3,24$ & 331.2 & 8.1 & 1.1 & 59 \\
\hline $36-5,24$ & 334.2 & 7.8 & 0.9 & 58 \\
\hline $37-1,24$ & 337.7 & 8.5 & 0.8 & 64 \\
\hline $37-3,18$ & 340.7 & 8.7 & 0.7 & 67 \\
\hline $37-5,13$ & 343.6 & 8.9 & 0.6 & 69 \\
\hline $38-1,1$ & 347.0 & 8.2 & 0.7 & 62 \\
\hline $38-3,18$ & 350.2 & 10.0 & 0.5 & 79 \\
\hline $38-5,20$ & 353.2 & 10.2 & 0.3 & 82 \\
\hline $39-3,21$ & 359.7 & 9.7 & 0.4 & 77 \\
\hline $39-5,24$ & 362.7 & 9.9 & 0.3 & 80 \\
\hline $40-1,15$ & 366.2 & 7.4 & 0.5 & 57 \\
\hline
\end{tabular}

TABLE 1 - Continued

\begin{tabular}{ccrcc}
\hline $\begin{array}{c}\text { Core, Section } \\
\text { Top of Interval } \\
(\mathrm{cm})\end{array}$ & $\begin{array}{c}\text { Subbottom } \\
\text { Depth } \\
(\mathrm{m})\end{array}$ & $\begin{array}{c}\text { Total } \\
\text { Carbon } \\
(\%)\end{array}$ & $\begin{array}{c}\text { Organic } \\
\text { Carbon }\end{array}$ & $\mathrm{CaCO}_{3}$ \\
\hline $40-3,24$ & 369.2 & 7.3 & 0.5 & 57 \\
$40-5,31$ & 372.3 & 10.0 & 0.4 & 80 \\
$41-3,20$ & 378.7 & 10.9 & 0.2 & 89 \\
$41-5,23$ & 381.7 & 9.8 & 0.4 & 78 \\
$42-1,24$ & 385.2 & 8.1 & 0.5 & 64 \\
$42-3,24$ & 388.2 & 7.1 & 0.4 & 56 \\
$42-5,50$ & 391.5 & 9.7 & 0.3 & 78 \\
$44-1,0$ & 404.0 & 9.9 & 0.3 & 80 \\
$44-2,24$ & 405.7 & 9.5 & 0.3 & 77 \\
$44-3,24$ & 407.2 & 9.9 & 0.3 & 80 \\
$45-1,124$ & 414.7 & 12.0 & 0.2 & 99 \\
$45-1,10$ & 413.6 & 12.0 & 0.1 & 99 \\
$45-5,18$ & 419.7 & 11.9 & 0.2 & 98
\end{tabular}

Site $\mathbf{2 6 3}$

\begin{tabular}{lrrrr}
$1-1,115$ & 1.2 & 6.8 & 0.4 & 53 \\
$2-1,103$ & 53.5 & 4.6 & 0.4 & 35 \\
$2-3,24$ & 55.7 & 9.5 & 0.1 & 78 \\
$3-1,24$ & 90.7 & 9.9 & 0.1 & 82 \\
$3-3,24$ & 93.7 & 10.3 & 0.2 & 85 \\
$3-5,24$ & 96.7 & 10.4 & 0.1 & 86 \\
$4-1,24$ & 109.7 & 2.0 & 0.5 & 12 \\
$4-3,24$ & 112.7 & 1.8 & 0.6 & 10 \\
$4-5,29$ & 115.8 & 1.6 & 0.5 & 9 \\
$5-1,130$ & 129.8 & 1.5 & 0.6 & 7 \\
$6-1,110$ & 148.6 & 1.5 & 0.6 & 7 \\
$6-3,24$ & 150.7 & 1.0 & 0.8 & 1 \\
$6-5,25$ & 153.8 & 1.1 & 0.9 & 2 \\
$7-1,24$ & 176.2 & 2.2 & 0.7 & 12 \\
$7-3,39$ & 179.4 & 1.0 & 0.7 & 2 \\
$8-1,63$ & 205.1 & 0.8 & 0.7 & 1 \\
$9-1,70$ & 224.2 & 0.7 & 0.7 & 0 \\
$9-3,22$ & 226.7 & 0.8 & 0.7 & 1 \\
$10-1,60$ & 243.1 & 0.6 & 0.5 & 1 \\
$10-3,9$ & 245.6 & 0.7 & 0.7 & 0 \\
$11-1,24$ & 261.7 & 1.5 & 0.4 & 9 \\
$11-3,27$ & 264.8 & 1.8 & 0.4 & 12 \\
$12-1,15$ & 280.7 & 0.6 & 0.6 & 1 \\
$13-1,56$ & 300.1 & 1.0 & 0.8 & 2 \\
$13-3,49$ & 303.0 & 0.2 & 0.5 & 0 \\
$13-3,23$ & 302.7 & 0.7 & 0.7 & 0 \\
$13-5,93$ & 306.4 & 0.7 & 0.7 & 0 \\
$14-1,12$ & 318.6 & 0.6 & 0.6 & 0 \\
$14-3,105$ & 322.6 & 0.5 & 0.6 & 0 \\
$14-5,49$ & 325.0 & 0.5 & 0.5 & 0 \\
$15-1,32$ & 337.8 & 0.4 & 0.4 & 0 \\
$15-3,26$ & 340.8 & 0.3 & 0.2 & 0 \\
$16-1,23$ & 356.7 & 0.4 & 0.3 & 0 \\
$17-1,63$ & 385.6 & 0.8 & 0.8 & 0 \\
$17-3,10$ & 388.1 & 0.8 & 0.8 & 0 \\
$17-5,88$ & 391.9 & 1.3 & 1.2 & 0 \\
$18-1,65$ & 414.2 & 0.9 & 0.9 & 0 \\
$18-3,20$ & 416.7 & 1.9 & 1.8 & 1 \\
$18-5,10$ & 419.6 & 1.5 & 1.4 & 1 \\
$19-1,143$ & 452.9 & 0.9 & 0.9 & 0 \\
$19-3,60$ & 455.1 & 0.9 & 0.9 & 0 \\
$19-5,8$ & 457.6 & 1.1 & 1.0 & 1 \\
$20-1,50$ & 480.5 & 1.0 & 1.0 & 0 \\
$20-3,70$ & 483.7 & 0.8 & 0.8 & 0 \\
$20-5,28$ & 486.3 & 1.1 & 1.0 & 0 \\
$21-1,0$ & 518.0 & 0.8 & 0.8 & 0 \\
$21-3,103$ & 522.0 & 1.9 & 1.6 & 2 \\
$21-5,12$ & 524.1 & 1.5 & 1.2 & 2 \\
$22-1,19$ & 556.2 & 1.6 & 1.2 & 3 \\
$22-2,18$ & 557.7 & 8.2 & 0.6 & 63 \\
$23-1,52$ & 594.5 & 9.7 & 0.3 & 78 \\
$23-3,23$ & 597.2 & 2.2 & 1.6 & 5 \\
\hline & & & &
\end{tabular}


G. W. BODE

TABLE 1 - Continued

\begin{tabular}{lcccc}
\hline $\begin{array}{c}\text { Core, Section } \\
\text { Top of Interval } \\
(\mathrm{cm})\end{array}$ & $\begin{array}{c}\text { Subbottom } \\
\text { Depth } \\
(\mathrm{m})\end{array}$ & $\begin{array}{c}\text { Total } \\
\text { Carbon } \\
(\%)\end{array}$ & $\begin{array}{c}\text { Organic } \\
\text { Carbon }\end{array}$ & $\mathrm{CaCO}_{3}$ \\
\hline $23-5,118$ & 601.2 & 1.7 & 1.6 & 1 \\
$24-1,139$ & 633.4 & 2.2 & 2.1 & 1 \\
$24-3,0$ & 635.0 & 1.8 & 1.7 & 1 \\
$24-5,36$ & 638.4 & 0.8 & 0.8 & 0 \\
$25-3,26$ & 673.3 & 1.4 & 1.4 & 1 \\
$26-3,25$ & 701.8 & 2.0 & 1.3 & 6 \\
\hline
\end{tabular}

TABLE 1 - Continued

\begin{tabular}{lcccc}
\hline $\begin{array}{c}\text { Core, Section } \\
\text { Top of Interval } \\
(\mathrm{cm})\end{array}$ & $\begin{array}{c}\text { Subbottom } \\
\text { Depth } \\
(\mathrm{m})\end{array}$ & $\begin{array}{c}\text { Total } \\
\text { Carbon } \\
(\%)\end{array}$ & $\begin{array}{c}\text { Organic } \\
\text { Carbon }\end{array}$ & $\mathrm{CaCO}_{3}$ \\
\hline & & & & \\
$26-5,115$ & 705.7 & 9.7 & 0.5 & 77 \\
$28-1,0$ & 727.0 & 7.7 & 0.7 & 59 \\
$28-1,35$ & 727.4 & 2.0 & 1.8 & 1 \\
$28-3,136$ & 731.4 & 0.7 & 0.7 & 0 \\
$29-1,135$ & 737.9 & 2.1 & 1.8 & 3 \\
$29-3,8$ & 739.6 & 2.1 & 1.9 & 2 \\
\hline
\end{tabular}

